\title{
Relationship between Erectile Dysfunction and Metabolic Syndrome in Patients at a University Hospital
}

\author{
Marina Gaglianone Teodoro da Silva ${ }^{1}$, Luis César Fava Spessoto ${ }^{2}$, \\ Fernando Nestor Fácio Junior ${ }^{2}$
}

${ }^{1}$ Undergraduate Student, São José Rio Preto School of Medicine (FAMERP), Brazil

${ }^{2}$ Department of Urology, São José Rio Preto School of Medicine (FAMERP), Brazil

\begin{abstract}
Introduction: Erectile dysfunction (ED) is the most common cause of sexual dysfunction in men and shares several risk factors with systemic conditions, such as cardiovascular disease and metabolic syndrome (MetS). Objective: Investigate the relationship between ED and MetS among patients at a university hospital. Patients and Methods: Patients with ED were retrospectively investigated in a cross-sectional study conducted at a university hospital. The following variables were analyzed: systemic arterial hypertension, type 2 diabetes mellitus (DM2), dyslipidemia, obesity, smoking, alcohol use, low adherence to treatment or ineffective treatment, medications, glycated hemoglobin and lipids (total cholesterol, HDL and triglycerides). Results: Among the 96 patients studied, $23(24 \%)$ met the criteria for MetS. Analyzing only one comorbidity, 10.4\% of patients had hypertension, 5.2\% had DM2 and 5.2\% had dyslipidemia. Age ranged from 21 to 78 years (mean: $56.9 \pm 12.2$ years) and 52\% of patients were older than 60 years of age. Abnormal glycated hemoglobin was found in $40.6 \%$ ) of patients; $41.6 \%$ had dyslipidemia; $47.9 \%$ had hypertension; and 39.6\% used anti-hypertensive medications. Smoking was found in $39.5 \%$ and alcohol use was found in $30.2 \%$. The results of the chi-squared test revealed that smoking was significantly associated with hypertension, DM2 and dyslipidemia $(\mathrm{P}<0.01)$. Nearly half $(46.9 \%)$ of the patients had low adherence or underwent ineffective treatment. Conclusion: No association was found between erectile dysfunction and metabolic syndrome. Significant associations were found between smoking and hypertension, DM2 and dyslipidemia.
\end{abstract}

\section{Introduction}

In the last ten years, male sexual dysfunction has become a topic of considerable interest among clinicians, researchers and public health administers. However, this condition involves multiple aspects that often give rise to questions and doubts on the part of both health professionals and patients. ${ }^{1}$

Erectile dysfunction (ED) is a common clinical condition that exerts a negative impact on quality of life. ${ }^{2}$ At a conference promoted by the US National Institutes of Health $(\mathrm{NIH})$ with the aim of redefining these different conditions, the term "sexual impotence" was replaced with ED to designate the inability for a man to achieve and maintain a sufficient erection for the performance of satisfactory sexual activity. ${ }^{3}$ Diverse physical and psychological components are involved in normal erectile function, including hormonal, neurological and vascular factors as well as aspects related to the corpora cavernosa. $^{4,5}$
ED affects 5 to $20 \%$ of men throughout the world and metabolic syndrome (MetS) has been found to be a risk factor for this condition. ${ }^{6-8}$ MetS is a disease that occurs mainly due to the global epidemic of obesity and emerges with insulin resistance, arterial hypertension, dyslipidemia and diabetes. Various definitions of MetS are found in the literature, but the presence of three or more of the conditions cited classifies the syndrome. ${ }^{9}$ The prevalence of MetS is high throughout the world, with rates of $9.5 \%$ reported for Europe and 35 to $39 \%$ for the USA. ${ }^{10}$ According to the US National Cholesterol Education Program, the criteria for the diagnosis of MetS are the presence of at least three of the following: abdominal circumference $>102 \mathrm{~cm}$ in men and $>88$ $\mathrm{cm}$ in women; plasma glucose $\geq 110 \mathrm{mg} / \mathrm{dL}$; triglycerides $\geq 150 \mathrm{mg} / \mathrm{dL} ; \mathrm{HDL}$ cholesterol $<40$ $\mathrm{mg} / \mathrm{dL}$ in men and $<50 \mathrm{mg} / \mathrm{dL}$ in women; systolic blood pressure $\geq 130 \mathrm{mmHg}$ or diastolic blood pressure $\geq 85 \mathrm{mmHg}^{11}$

A normal erection is a hemodynamic event that involves both the central nervous system and local

This article is published under the terms of the Creative Commons Attribution License 4.0

Author(s) retain the copyright of this article. Publication rights with Alkhaer Publications.

Published at: http://www.ijsciences.com/pub/issue/2019-06/

DOI: 10.18483/ijSci.2083; Online ISSN: 2305-3925; Print ISSN: 2410-4477 
systems and is regulated by the relaxation of the cavernous arteries and the smooth muscles of the corpora cavernosa. ${ }^{12}$ The relaxation of the smooth muscles of the corpora cavernosa and the erection of the penis depend on the balance between vasoconstricting and vasodilating factors. ${ }^{13,14}$ In ED, an imbalance occurs between contraction and relaxation of the smooth muscles of the corpora cavernosa. $^{15,16}$

ED is associated with a number of risk factors, such as age, diabetes mellitus, arterial hypertension, vascular disease, vascular surgery, coronary artery disease, pelvic or penile trauma, radical pelvic surgery, high levels of cholesterol, low levels of high-density lipoprotein (HDL), drug use, neurological diseases (spinal cord injury, multiple sclerosis and stroke), Peyronie's disease, priapism, radiotherapy, depression, alcohol intake, a lack of sexual knowledge and an inadequate interpersonal relationship. ${ }^{15-18}$

Smoking is the major isolated modifiable cardiovascular risk factor in the world population. ${ }^{19}$ Cigarettes alone double the probability of cardiovascular disease and this risk is quadrupled when smoking is concomitant to dyslipidemia or arterial hypertension.

The precise identification of potential risk factors is essential to the prevention of ED and the determination of the association between ED and vascular disease. ${ }^{20,21}$ The most common organic cause of ED is likely the consequence of vascular (arterial or venous) abnormalities, which are often associated with atherosclerosis and diabetes mellitus. ${ }^{19,22}$ Atherosclerosis is a risk factor for ED in $70 \%$ of men aged 60 years or older in the United States. ${ }^{23}$ Systemic arterial hypertension alone has a negative impact on erectile function in nearly all hypertensive men. ${ }^{24}$ Anti-hypertensive agents, such as alpha methyldopa, clonidine, reserpine, propranolol and other beta-adrenergic agents, hydralazine and diuretics cause sexual problems ranging from a diminished libido to ED in 5 to $80 \%$ of cases. ${ }^{25}$ It is likely that the normalization of mild arterial tension leads to a reduced functional blood flow, which hinders erection. ${ }^{26}$

As the prevalence of ED in patients with MetS is twofold higher compared to control individuals, ${ }^{27}$ the present study is justified from the clinical viewpoint by the possibility of ED worsening in patients with MetS. Therefore, the determination of a possible association between ED and MetS can contribute to improving the treatment of such patients.
The aim of the present study was to investigate the relationship between erectile dysfunction and metabolic syndrome in patients at a university hospital.

\section{Patients and Methods}

Patients with ED at the urology outpatient clinic of a university hospital in São José do Rio Preto, SP, Brazil, were evaluated retrospectively in a crosssectional study. This study received approval from the Human Research Ethics Committee of the Faculdade de Medicina de São José Rio Preto (FAMERP [São José Rio Preto School of Medicine]).

Data collection involved the analysis of the charts of patients with ED in the previous 12 months, considering clinical histories and laboratory exams in the period between August 2018 and January 2019. The diagnosis of ED was based on clinical data (signs and symptoms reported by the patients), following the protocol of the service, which is based on the International Index of Erectile Function (IIEF$5)$. The patients in the present study were classified only with regard to the presence of ED, independently of the degree of impairment.

The medical chart and laboratory exams [glycated hemoglobin and lipids (total cholesterol, HDL and triglycerides)] of each patient were analyzed. The laboratory exams were performed with standardized methods by experienced physicians and analyzed based on the reference values defined for the hospital. The patients were classified according to clinical data: 1) presence/absence of comorbidities, such as hypertension, type 2 diabetes mellitus (DM2), dyslipidemia and obesity; 2) daily habits, such as smoking and alcohol use; and 3) low adherence to treatment or ineffective treatment. Medications were also analyzed. Smokers and exsmokers were placed in the same group. The same was done for alcohol users and ex-alcohol users. Low adherence to treatment or ineffective treatment was judged based on the clinical history and the analysis of abnormal results on the laboratory exams in comparison to the reference values proposed by the service in the study period.

Statistical analysis involved the calculation of prevalence coefficients and the chi-square test considering a $1 \%$ significance level and one degree of freedom. The aim of the statistical analysis was to demonstrate the influence of smoking on the presence/absence of comorbidities (hypertension, DM2 and dyslipidemia).

\section{Results}

The prevalence indices revealed that 23 of the 96 patients $(24 \%)$ had the criteria for the diagnosis of 
metabolic syndrome. Analyzing only one comorbidity, $10.4 \%$ of patients had hypertension alone, 5.2\% had DM2 alone and 5.2\% had dyslipidemia alone. Age ranged from 21 to 78 years (mean: $56.9 \pm 12.2$ years) and $52 \%$ of patients were older than 60 years of age. Abnormal glycated hemoglobin was found in $40.6 \%$ of patients, $41.6 \%$ had dyslipidemia and $47.9 \%$ had hypertension. The use of beta-blockers was found in $25 \%$ of the patients with ED and the use of diuretics was found in $14.6 \%$, totaling $39.6 \%$ of patients using antihypertensive medications. Smoking was found in $39.5 \%$, alcohol use was found in $30.2 \%$ and the combined use of tobacco and alcohol was found in $25 \%$. The results of the chi-squared test revealed that smoking was significantly associated $(\mathrm{P}<0.01)$ with hypertension, DM2 and dyslipidemia (Table 1).

Table 1 - Association between smoking and comorbidities*

\begin{tabular}{lccc}
\hline & With comorbidities & $\begin{array}{c}\text { Without } \\
\text { comorbidities }\end{array}$ & Total \\
\hline Smokers & 31 & 7 & 38 \\
Non-smokers & 31 & 27 & 58 \\
Total & 62 & 34 & 96 \\
\hline$*$ chi-squared test & & &
\end{tabular}

Nearly half $(46.9 \%)$ of the patients had low adherence or underwent ineffective treatment.

\section{Discussion}

The mean age of the present sample was $56.9 \pm 12.2$ years, which confirms the direct association between ED and ageing. ${ }^{28}$ The severity of the condition was not determined in this study due to the lack of documentation of IIEF values on the patient charts.

From the endocrinological standpoint, MetS or at least one of its components was highly prevalent, accounting for $44.8 \%$ of the patients with ED in the present study, which is somewhat higher than the $33.8 \%$ prevalence reported by the American Association of Urology. These data offer further evidence of the influence of comorbidities of MetS on the physiopathology of ED as well as the aggravation of this condition with the greater number of associated comorbidities $(5.2 \%$ increase in the prevalence of ED with each individual comorbidity and a $24 \%$ increase with MetS). ${ }^{6}$

Another important finding was the high glycated hemoglobin in comparison to the reference value in $40.6 \%$ of the patients. This finding lends support to the hypothesis that vascular microinjuries stemming from hyperglycemia are related to difficulty in achieving an erection due to the glycosylation of the cavernous tissue of the penis, which leads to impaired collagen turnover and subsequent ED. ${ }^{29}$

Regarding anti-hypertensive medications, only betablockers and diuretics were investigated in the present study and were associated with ED, as 39.6\% of the patients made use of these types of drugs. This finding suggests the possibility that ED is a side effect of such medications, as discussed in previous studies. $^{8}$

Lifestyle interventions have been shown to be important to the regression of ED and the better control of cardiovascular risk. Indeed, lifestyle changes are recommended by the European Association of Urology as complementary treatment for $\mathrm{ED}^{30}$ Studies have demonstrated significant improvements in ED with the interruption of smoking in young patients with no associated comorbidities. Among the smokers with ED in the present study, only $18.4 \%$ did not have comorbidities, which suggests that smoking exerts a strong influence on the predisposition to comorbidities and the consequent development of ED.

In this series, MetS was prevalent in the physiopathology of ED, especially the glycemic component, as high glycated hemoglobin was found in nearly half of the patients. Such lack of glucose control is often the result of non-adherence to treatment or dubious treatment.

The association with ageing is undeniable, but factors such as tobacco and alcohol use can aggravate ED as well as its associated comorbidities. Indeed, ED is more prevalent in individuals who have at least one component of MetS and also smoke and/or drink.

Among the present findings, it should be stressed that medications, such as beta-blockers for patients with hypertension, can aggravate ED. Finally, based on the literature, the prevalence of ED was within the expected range for the profile of the sample. 


\section{Conclusion}

In the present study, no significant association was found between erectile dysfunction and metabolic syndrome. Significant associations were found between smoking and hypertension, DM2 and dyslipidemia.

\section{Acknowledgements}

The authors are grateful to the Brazilian fostering agency Conselho Nacional de Desenvolvimento Científico e Tecnológico (CNPq [National Council of Scientific and Technological Development]).

\section{References}

1. Montorsi P, Ravagnani PM, Vlachopoulos C. Clinical significance of erectile dysfunction developing after acute coronary event: exception to the rule or confirmation of the artery size hypothesis? Asian J Androl 2015;17(1):21-5.

2. Shamloul R, Ghanem H. Erectile dysfunction. Lancet. 2013;381:153-165.

3. NIH Consensus Conference. Impotence. NIH Consensus Development Panel on Impotence. JAMA. 1993;270(1):8390.

4. Melman A. Evaluation and management of erectile dysfunction. Surg Clin North Am 1988;68(5):965-81.

5. Mulcahy JJ. Current approach to the treatment of penile implant infections. Ther Adv Urol 2010;2(2):69-75.

6. Heidler $\mathrm{S}$, et al. Is the metabolic syndrome an independent risk factor for erectile dysfunction? J Urol 2007;177:651654.

7. Jackson G. The metabolic syndrome and erectile dysfunction: multiple vascular risk factors and hypogonadism. Eur Urol 2006;50:426-427.

8. Kubin M, Wanger G, Fugl-Meyer AR. Epidemiology of erectile dysfunction. Int J Impot Res 2003;15:63-71.

9. Expert Panel on Detection, E. and Treatment \& Adults, o. H. B. C. i Executive Summary of The Third Report of The National Cholesterol Education Program (NCEP) Expert Panel on Detection, Evaluation, and Treatment of High Blood Cholesterol in Adults (Adult Treatment Panel III). JAMA 2001;285:2486-2497.

10. Golden SH, Robinson KA, Saldanha I, Anton B, Ladenson PW. Clinical review: Prevalence and incidence of endocrine and metabolic disorders in the United States: a comprehensive review. J Clin Endocrinol Metab 2009; 94:1853-1878.

11. Santos Ce, Schrank Y, Kupfer R. Análise crítica dos critérios da OMS, IDF e NCEP para síndrome metabólica em pacientes portadores de diabetes melito tipo 1. Arq Bras Endocrinol Metab 2009;53(9):1096-1102.

12. Kapur V, Schwarz ER. The relationship between erectile dysfunction and cardiovascular disease. Part I: pathophysiology and mechanisms. Rev Cardiovasc Med 2007;8(4):214-9.

13. El-Sakka AI, Morsy AM, Fagih BI. Severity of erectile dysfunction could predict left ventricular diastolic dysfunction in patients without overt cardiac complaint. J Sex Med 2011;8(9):2590-7.

14. Sivalingam S, Hashim H, Schwaibold H. An overview of the diagnosis and treatment of erectile dysfunction. Drugs 2006;66(18):2339-55.
15. El-Sakka AI, Morsy AM, Fagih BI, Nassar AH. Coronary artery risk factors in patients with erectile dysfunction. J Urol 2004;172(1):251-4.

16. Francavilla S, Bocchio M, Pelliccione F, Necozione S, Francavilla F. Vascular aetiology of erectile dysfunction. Int J Androl 2005;28 Suppl 2:35-9.

17. Brunner H, Cockcroft Jr, Deanfield J, Donald A, Ferrannini E, Halcox J, et al. Endothelial function and dysfunction. Part II: Association with cardiovascular risk factors and diseases. A statement by the Working Group on Endothelins and Endothelial Factors of the European Society of Hypertension. J Hypertens 2005;23(2):233-46.

18. Gazzaruso C, Giordanetti S, De Amici E, Bertone G, Falcone C, Geroldi D, et al. Relationship between erectile dysfunction and silent myocardial ischemia in apparently uncomplicated type 2 diabetic patients. Circulation 2004;110(1):22-6.

19. Mulhall J, Teloken P, Brock G, Kim E. Obesity, dyslipidemias and erectile dysfunction: a report of a subcommittee of the sexual medicine society of North America. J Sex Med 2006;3(5):778-86.

20. Montorsi P, Ravagnani PM, Galli S, Rotatori F, Veglia F, Briganti A, et al. Association between erectile dysfunction and coronary artery disease. Role of coronary clinical presentation and extent of coronary vessels involvement: the COBRA trial. Eur Heart J 2006;27(22):2632-9.

21. Sekoranja L, Bianchi-Demicheli F, Gaspoz JM, Mach F. Erectile dysfunction: a potential useful marker for cardiovascular disease. Rev Med Suisse 2006;2(58):774-6, 78.

22. Gupta BP, Murad MH, Clifton MM, Prokop L, Nehra A, Kopecky SL. The effect of lifestyle modification and cardiovascular risk factor reduction on erectile dysfunction: a systematic review and meta-analysis. Arch Intern Med 2011;171(20):1797-803.

23. Canat L, Cicek G, Atis G, Gurbuz C, Caskurlu T. Is there a relationship between severity of coronary artery disease and severity of erectile dysfunction? Int Braz J Urol 2013;39(4):465-73.

24. Sai Ravi Shanker A, Phanikrishna B, Bhaktha Vatsala Reddy C. Association between erectile dysfunction and coronary artery disease and its severity. Indian Heart J 2013;65(2):180-6.

25. Meller SM, Stilp E, Walker CN, Mena-Hurtado C. The link between vasculogenic erectile dysfunction, coronary artery disease, and peripheral artery disease: role of metabolic factors and endovascular therapy. J Invasive Cardiol 2013;25(6):313-9.

26. Spessoto LC, Cordeiro JA, Godoy JMG. Effect of systemic arterial pressure on erectile dysfunction in the initial stages of chronic arterial insufficiency. BJU Int 2010;11:1723-5.

27. Kaya E, Sikka SC, Gur S. A comprehensive review of metabolic syndrome affecting erectile dysfunction. J Sex Med 2015;12:856-875.

28. Sanchez-Cruz, J. J., Cabrera-Leon, A., Martın-Morales, A., Fernandez, A., Burgos, R., Rejas, J. Male erectile dysfunction and health-related quality of life. Eur Urol 2003;44: 245-253.

29. Sanjay S, Bharti GS, Manish G, Rajeev, P, Pankaj, A, Puspalata, A, et al. Metabolic syndrome: An independent risk factor for erectile dysfunction. Indian $\mathrm{J}$ Endocrinol Metab 2015;19(2):277-82.

30. Hatzimouratidis, K., Giuliano, F., Moncada, I., Muneer, A. Salonia, A., Verze, P. EAU guidelines on erectile dysfunction, premature ejaculation, penile curvature and priapism. Eur Assoc Urol 2016;46. 